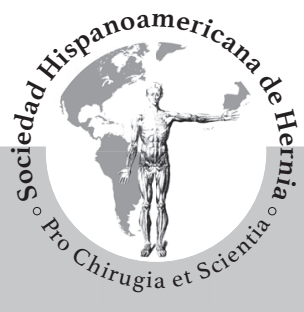

\title{
Reacción a cuerpo extraño tras reparación de hernia inguinal y alteración autoinmune tipo esclerodermia
} Foreign body reaction after inguinal hernia repair

\section{Alfredo Moreno-Egea ${ }^{1,2}$, Alfredo Moreno Latorre ${ }^{3}$}

1Jefe, Clínica Hernia. Hospital Universitario La Vega. Murcia (España). ²Profesor de Anatomía Quirúrgica. Departamento de Anatomía Humana. Facultad de Medicina. Universidad Católica San Antonio (UCAM). Murcia (España). ${ }^{3}$ Residente de MFC. Servicio de Urgencias. Hospital Santa Lucía. Cartagena, Murcia (España)

Recibido: 14-01-2018

Aceptado: 05-02-2018

\section{Palabras clave:}

Reacción a cuerpo extraño, malla, hernioplastia inguinal, esclerodermia, enfermedad autoinmune.

Key words:

Foreign body reaction, mesh, inguinal hernia repair, scleroderma, autoinmune disease.

\section{Resumen}

Introducción: El uso de una malla en la reparación de la hernia inguinal tiene el riesgo de rechazo tardío o de complicaciones infecciosas relacionadas con el cuerpo extraño implantado. El objetivo de este estudio es describir un caso de rechazo crónico asociado a una esclerodermia.

Caso clínico: Se describe el caso de una mujer de 72 años que tras una hernioplastia con malla de polipropileno desarrolla una enfermedad autoinmune progresiva e invalidante. Se realiza extirpación del material extraño por vía anterior. No hubo morbilidad y no se han presentado complicaciones durante un seguimiento de 6 meses.

Discusión: La malla que se implanta en una hernioplastia inguinal puede generar una reacción a cuerpo extraño y una enfermedad de tipo autoinmune como la esclerodermia.

\footnotetext{
Abstract

Background: The use of a mesh in inguinal hernia repair caries the risk of late rejection or infectious complications related to the mesh. The aim of this study was to describe a case associated with scleroderma.

Case report: We describe the case of a 72-year-old woman who, after hernioplasty with a polypropylene mesh, develops a progressive and disabling autoimmune disease. Extirpation of the foreign material is carried out anteriorly. There was no morbidity and there were no complications during a 6-month follow-up.

Discussion: The mesh that is implanted in an inguinal hernioplasty can generate a reaction to a foreign body and an autoimmune type disease such as scleroderma.
}

\footnotetext{
* Autor para correspondencia: Alfredo Moreno-Egea. Clínica Hernia. Hospital Universitario La Vega. c/ Dr. Román Alberca, s/n. 30008 Murcia (España) Correo electrónico: morenoegeaalfredo@gmail.com
} 


\section{Introducción}

Las mallas han supuesto un innegable beneficio en la cirugía de las hernias. Gracias a ellas, las operaciones se han simplificado desde un punto de vista técnico y las tasas de recidivas de cualquier cirujano han descendido de forma notable ${ }^{1-3}$. Pero no debemos olvidar que las mallas no dejan de ser un material extraño que es implantado en un organismo vivo y que este responde con una reacción de integración y curación. El problema se presenta cuando este proceso no es controlado y se genera una respuesta inmune anormal. Estos casos, deben ser excepcionales, pero cuando hablamos de una operación que implica a miles de personas al año, al final, esta posibilidad de complicación no debe ser tan despreciable.

En la literatura se han descrito complicaciones de la malla por erosión, migración, infección y rechazo, pero no se ha documentado su asociación con enfermedades autoinmunes o del tejido conectivo (como el reumatismo, lupus o esclerodermia) ${ }^{4-11}$. El objetivo de este trabajo es documentar el primer caso de una paciente operada de una hernia y complicada con una esclerodermia.

\section{Caso clínico}

Mujer de 72 años, sin alergias ni enfermedades conocidas. IMC de $24 \mathrm{~kg} / \mathrm{m}^{2}$. Cuarenta y siete años de matrimonio y 4 hijos por parto natural sanos. No operaciones previas. No tratamientos crónicos. Deportista profesional. De forma rutinaria, en un chequeo anual, le detectan por ecografía una hernia inguinal asintomática. Consulta al cirujano que le aconseja cirugía. Se opera en enero de 2016, sin incidencias mencionadas. Tras la cirugía, la salud de la paciente va empeorando de forma progresiva. Se queja en varias ocasiones de un bulto duro a nivel de su ingle, con diferente sensibilidad hasta la parte anterior del músculo pero sin signos de infección. Unos días después le aparece en el antebrazo derecho una mancha ovalada por la que consulta al dermatólogo, se realiza una biopsia y se diagnostica de una esclerodermia localizada (morfea circunscrita). Le aconsejan no darle importancia. La siguiente semana aparecen dolores no conocidos en las muñecas, y posteriormente va generalizándose a rodillas, codos, hombros, tobillos y cervicales, con una sensación de debilidad creciente (no recuerda episodios anteriores tipo reumatoideos).

Consultada por varios especialistas, fue sometida a todo tipo de análisis y de pruebas: laboratorio completo con estudio inmune normal (IgG, anti-LA, anti-Smith, anti-Ro, Esclero 70, JO-1) solo Sm/RNP positivo $76(\mathrm{n}<12)$, ac. antinucleares $1 / 5120(\mathrm{n}<1 / 80)$ y FR 42 (n < 14); radiologías, tomografías, densitometrías, ecografías, etc., siendo todo normal. Densitometría con osteopenia leve en columna lumbar y cuello de fémur. Mapeo negativo para dolor neuropático. No entendía por qué había cambiado su vida de una forma tan rápida después de operarse de una hernia sin molestias previas. Todo lo refería a partir de esta operación y el tumor duro de su ingle seguía ahí.

Con la historia clínica de 1 año y medio de evolución se realiza un posible diagnóstico de rechazo autoinmune de la malla y enfermedad reumatoide se explica la situación a la paciente para solicitar su consentimiento para una nueva operación, con sus riesgos y posible ineficacia en resolver su estado evolutivo. Se opera por vía anterior, se encuentra una gran zona de adenopatías de gran tamaño sobre la ingle. Se realiza extirpación ganglionar para estudio anatomopatológico. Se abre el espacio inguinal y se extirpa la malla por completo, arrugada y plegada sobre el pubis. El espacio es cerrado mediante técnica de Shouldice (figs. 1-4). Se envían muestras para estudio anatomopatológico de la pieza, tejido inflamado y de la lesión del antebrazo. La paciente es alta a las $12 \mathrm{~h}$ sin problemas locales ni generales.

\section{Anatomía patológica}

El estudio histológico se informa como: a) tejido conectivo denso tipo aponeurosis con cavidades vacías de estructura tridimensional helicoidal, rodeadas por débil respuesta linfoide y cavidad quística formada por posible desgarro (tejido conectivo continente

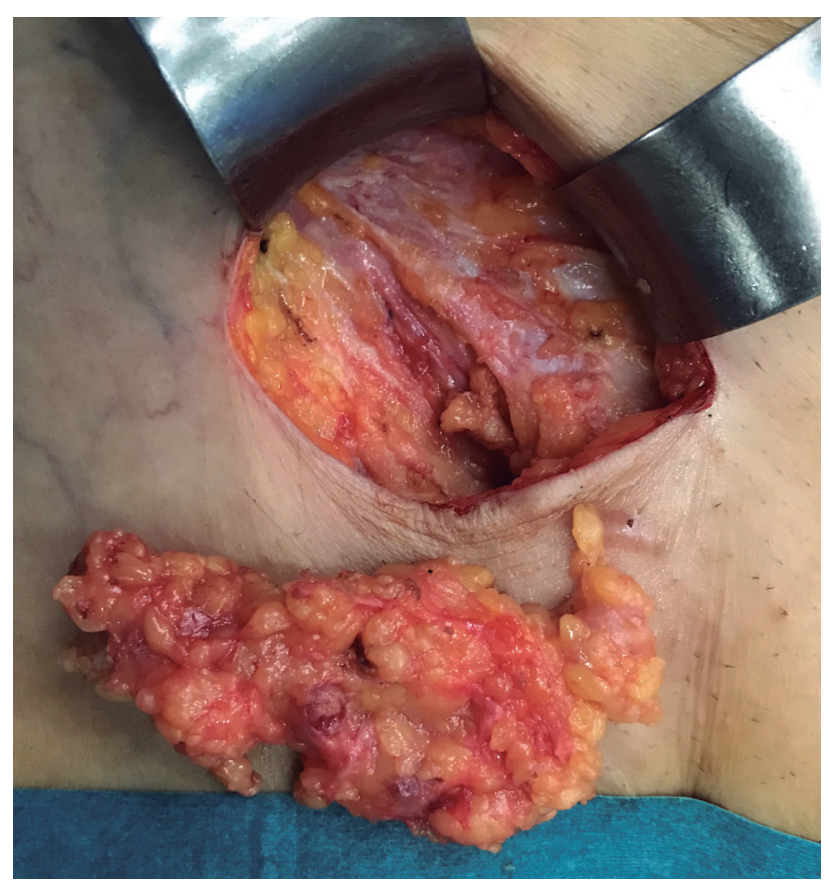

Figura 1. Detalle del tejido linfograso reactivo inguinal.

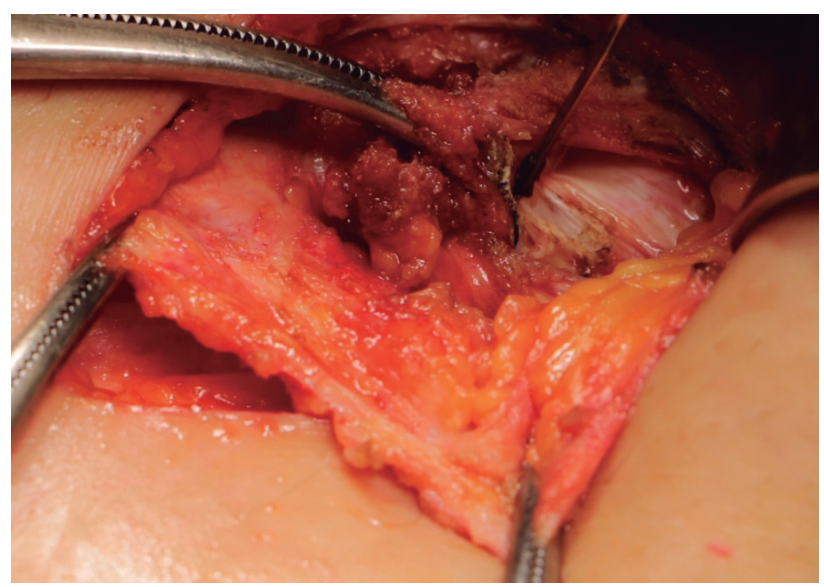

Figura 2. Extirpación de la malla implantada. 


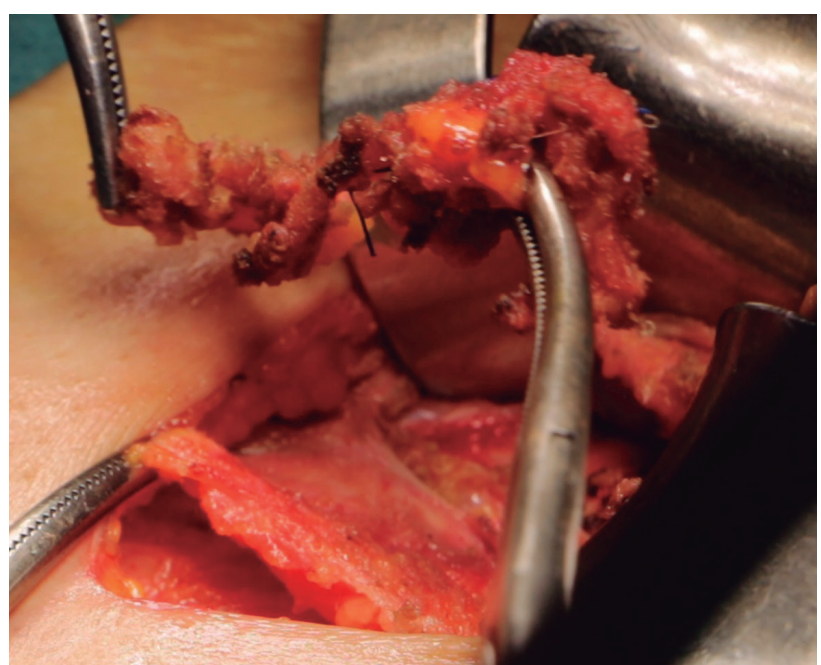

Figura 3. Malla totalmente extirpada de la región inguinal.

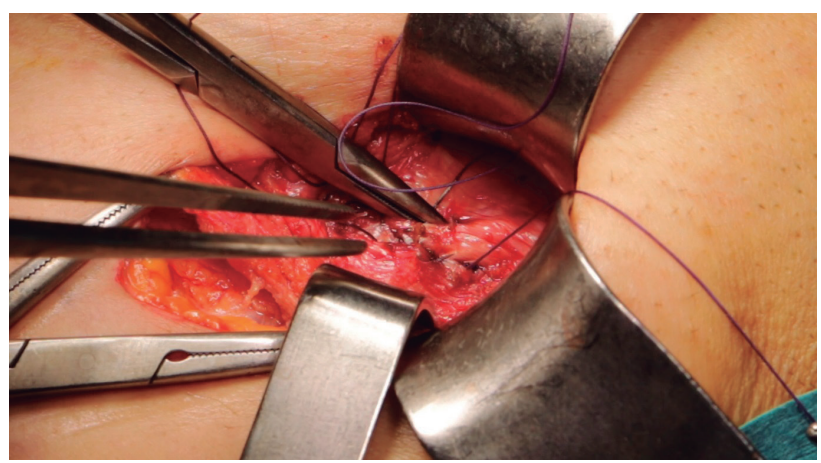

Figura 4. Reconstrucción mediante suturas de ida y vuelta.

de malla); b) ganglios con engrosamiento capsular y trabéculas, folículos hiperplásicos y abundantes células plasmáticas en las trabéculas (pseudotumor inflamatorio ganglionar); y c) epidermis con hiperpigmentación basal, dermis con haces de colágeno gruesos, esclerosis, horizontales que rodean ovillos sudoríparos sin adipocitos (morfea) (figs. 5-7).

\section{Evolución}

La paciente presenta mejoría clínica inmediata con recuperación funcional habitual. A las 2 semanas desaparece la lesión cutánea, solo persiste un engrosamiento fibroso al pinzamiento del brazo pero sin cambios en la piel. La movilidad es mejor y a las 4 semanas inicia ejercicios y retoma su actividad deportiva. No presenta dolor ni tumefacciones en articulaciones. La analítica de control ha normalizado sus parámetros inmunitarios (también los Sm/ RNP, ac. antinucleares y FR).

\section{Discusión}

Actualmente, los cirujanos no podemos predecir qué pacientes pueden desarrollar una reacción a la malla implantada durante

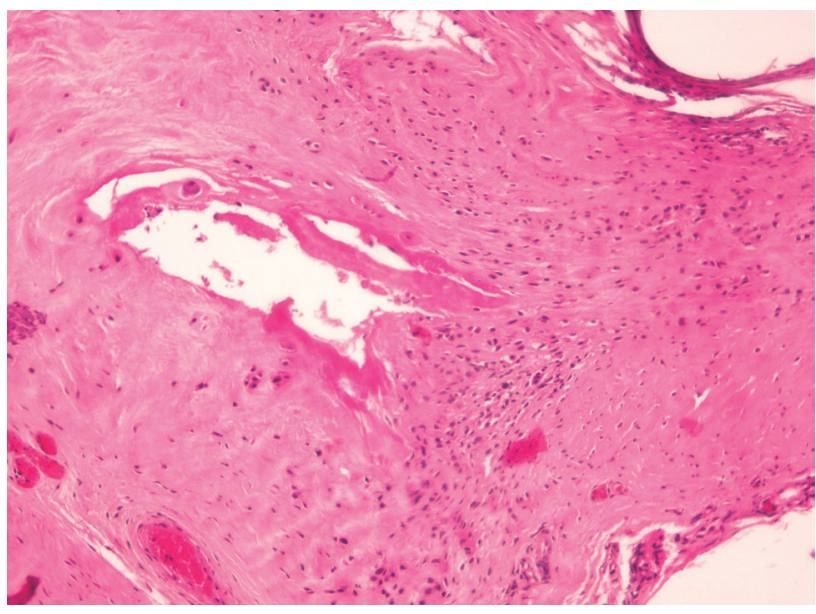

Figura 5. Microscopia de la entesitis y desgarro muscular. Tinción con hematoxilina-eosina (se ve también en un lado, a nivel de los dos ángulos, los huecos de la malla).

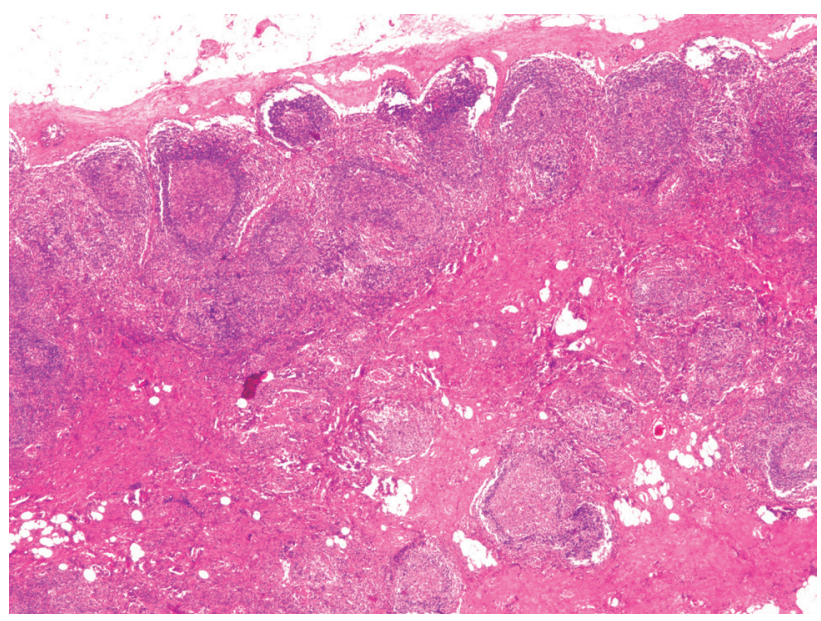

Figura 6. Microscopia de la afectación ganglionar. Tinción con hematoxilina-eosina.

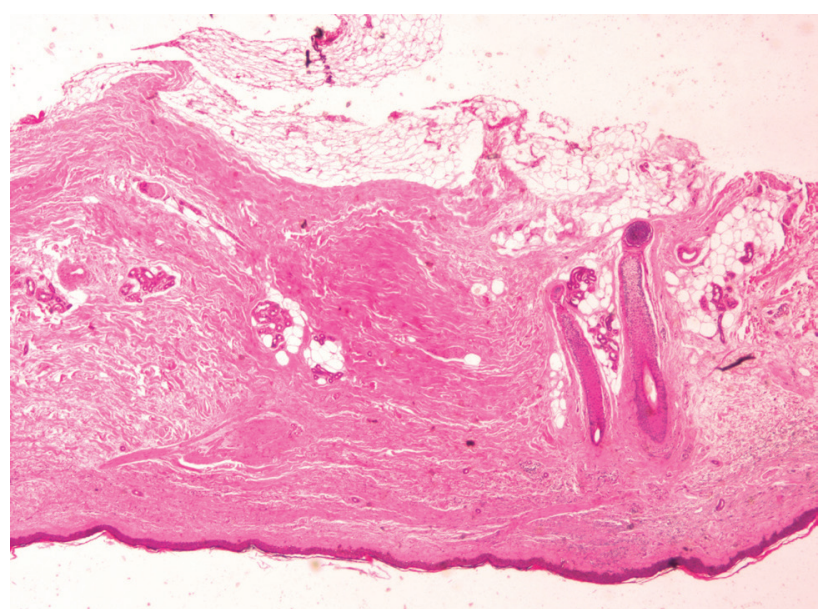

Figura 7. Microscopia de la morfea. Tinción con hematoxilina-eosina. Epidermis irregular, esclerosis e infiltrado inflamatorio linfoplasmocitario superficial y profundo perivascular. 
una hernioplastia inguinal. Todas las mallas generan, en cualquier medida, una reacción a cuerpo extraño controlada como parte del proceso normal de integración y curación ${ }^{1,2}$. Aunque se piensa que las mallas usadas en cirugía son inertes (no inmunogénicas), realmente ninguna es biológicamente inerte. Cualquier malla implantada es seguida de una respuesta de curación caracterizada por tres posibilidades: a) destrucción o lisis; b) inclusión o tolerancia; y c) rechazo. El sistema inmunitario reconoce la malla como un material extraño e intenta destruirla. El rechazo es impulsado principalmente por la respuesta inmune de los linfocitos $T$, células estimuladas por la presencia de un determinado antígeno, proliferan y atacan el material extraño.

Desde un punto de vista histológico, la interacción malla - organismo se caracteriza por tres puntos: el tamaño de la reacción del tejido, la densidad celular y la actividad fibroblástica. Cuando se altera este proceso normal con una prolongación de la reacción inflamatoria, se crea un acumulo adicional de fibroblastos con aumento de la fibrosis y una integración más rápida que conlleva dolor, retracción y rechazo de la malla. Esta reacción depende en gran medida de la naturaleza fisicoquímica de la malla (material, filamentos, tamaño de poro, etc. $)^{1,2}$. La consecuencia de este hecho es que el cirujano no puede olvidar que toda esta secuencia de acontecimientos posibles es iniciada por un gesto cotidiano que depende de él, la implantación de una malla, y así, la elección del tipo de material debe ser un paso totalmente razonado.

La mayoría de pacientes tienen una inmunidad normal y la implantación de una malla no genera ninguna complicación, pero en algunos casos, debemos aceptar la posibilidad de que puede originarse una respuesta inmune no controlada, de mayor intensidad y causar una enfermedad sistémica de tipo autoinmune: lupus, síndrome de Sjögren, esclerodermia, polimiositis, enfermedad mixta del tejido conectivo, poliarteritis nudosa o angeítis por hipersensibilidad. En 2016, Towfigh comunicó la posible asociación de una malla con una fibromialgia ${ }^{12}$. Esta es la primera referencia que se documenta de una hernioplastia seguida de una esclerodermia.

El diagnóstico de una complicación por reacción a cuerpo extraño no es sencillo. La clínica no es específica y, como en nuestro caso, la exploración inguinal puede ser prácticamente normal ( $\sin$ signos de inflamación). La presencia de síntomas generales, tipo gastrointestinal o urinario, dificultan aún más pensar en esta posibilidad. La historia de cirugía reciente y la progresiva afectación debilitante deben alertar sobre esta posibilidad. Algunos pacientes con problemas de tipo reumático o enfermedades del tejido conectivo podrían estar asociados a esta entidad y no encontrar un beneficio con ninguna terapia conservadora. Cuando la secuencia de acontecimientos es tan dramática como en nuestro caso, la extirpación de la malla por un cirujano especializado debería ser considerada como una parte más del plan de tratamiento.

Towfigh sugiere que los pacientes portadores de una hernia diagnosticados de fibromialgia o enfermedad autoinmune deberían ser considerados para reparación sin malla. Parece razonable limitar un poco el uso de las mallas en pacientes seleccionados con enfermedades sistémicas o autoinmunes, pacientes susceptibles de dolor crónico, de infecciones recurrentes, con enfermedad neoplásica, con inmunidad alterada, etc. Estos pacientes podrían ser remitidos a un especialista para que planteara una reparación de la pared abdominal sin material extraño o al menos, con el uso de mallas mejor seleccionados al caso (menor densidad, recubrimiento metálico, etc.). Quizás el futuro nos permita disponer de alguna prueba sencilla para que los cirujanos seamos capaces de detectar antes de la operación, el estado inmune del paciente y su respuesta a determinado tipo de malla, evitando así la posibilidad de complicación con una enfermedad autoinmune.

\section{Bibliografía}

1. Bellon JM. Revisión de una clasificación de materiales protésicos destinados a la reparación herniaria: correlación entre estructura y comportamiento en los tejidos receptores. Rev Hispanoam Hernia. 2014;02:49-57.

2. Baylón K, Rodríguez-Camarillo P, Elías-Zúñiga A, Díaz-Elizondo JA, Gilkerson R, Lozano K. Past, Present and Future of Surgical Meshes: A Review. Membranes (Basel). 2017;7(3).

3. Chaveli C, Blázquez L, Marzo P, González G, de Miguel M, Abadía $\mathrm{T}$, et al. Dolor crónico inguinal tras reparación herniaria. Análisis de nuestra serie a través de un cuestionario postal. Rev Hispanoam Hernia. 2013;1(1):5-11.

4. Viscido G, Biurrum J, Bocco MC, Olmedo I, Picón Molina H, Mandojana $\mathrm{F}$, et al. Infección protésica crónica asociada a extrusión tras hernioplastia umbilical. Rev Hispanoam Hernia. 2017;5(3):108-11.

5. Iakovlev V, Koch A, Petersen K, Morrison J, Grischkan D, Oprea V, et al. A Pathology of Mesh and Time: Dysejaculation, Sexual Pain, and Orchialgia Resulting From Polypropylene Mesh Erosion Into the Spermatic Cord. Ann Surg. 2017. DOI: 10.1097/ SLA.0000000000002134.

6. Seker D, Kulacoglu H. Long-term complications of mesh repairs for abdominal-wall hernias. J Long Term Eff Med Implants. 2011;21(3):205-18.

7. Jani K, Palanivelu C, Malladi V, Rajan PS, Rajapandian S, Shetty $\mathrm{R}$, et al. Late rejection after transabdominal pre-peritoneal inguinal repair: laparoscopic extraction of mesh. Indian J Gastroenterol. 2005;24(5):219-20.

8. Bodenbach M, Bschleipfer T, Stoschek M, Beckert R, Sparwasser C. Intravesical migration of a polypropylene mesh implant 3 years after laparoscopic transperitoneal hernioplasty. Urologe A. 2002;41(4):366-8.

9. Foschi D, Corsi F, Cellerino P, Trabucchi A, Trabucchi E. Late rejection of the mesh after laparoscopic hernia repair. Surg Endosc. 1998;12(5):455-7.

10. Hofbauer C, Andersen PV, Juul P, Qvist N. Late mesh rejection as a complication to transabdominal preperitoneal laparoscopic hernia repair. Surg Endosc. 1998;12(9):1164-5.

11. Al-Subaie S, Al-Haddad M, Al-Yaqout W, Al-Hajeri M, Claus C. A case of a colocutaneous fistula: A rare complication of mesh migration into the sigmoid colon after open tension-free hernia repair. Int J Surg Case Rep. 2015;14:26-9.

12. Jacob BP, Chen DC, Ramshaw B, Towfigh S, editors. The SAGES Manual of Groin Pain. 2016;33:429-34. 